\title{
COMMENTARY
}

\section{Predicting Alzheimer's risk: Why and how?}

\author{
Deborah E Barnes ${ }^{* 1-3}$ and Sei J Lee Le $^{4,5}$
}

\begin{abstract}
Because the pathologic processes that underlie Alzheimer's disease (AD) appear to start 10 to 20 years before symptoms develop, there is currently intense interest in developing techniques to accurately predict which individuals are most likely to become symptomatic. Several AD risk prediction strategies - including identification of biomarkers and neuroimaging techniques and development of risk indices that combine traditional and nontraditional risk factors - are being explored. Most AD risk prediction strategies developed to date have had moderate prognostic accuracy but are limited by two key issues. First, they do not explicitly model mortality along with $A D$ risk and, therefore, do not differentiate individuals who are likely to develop symptomatic AD prior to death from those who are likely to die of other causes. This is critically important so that any preventive treatments can be targeted to maximize the potential benefit and minimize the potential harm. Second, AD risk prediction strategies developed to date have not explored the full range of predictive variables (biomarkers, imaging, and traditional and non-traditional risk factors) over the full preclinical period (10 to 20 years). Sophisticated modeling techniques such as hidden Markov models may enable the development of a more comprehensive $A D$ risk prediction algorithm by combining data from multiple cohorts. As the field moves forward, it will be critically important to develop techniques that simultaneously model the risk of mortality as well as the risk of $A D$ over the full preclinical spectrum and to consider the potential harm as well as the benefit of identifying and treating high-risk older patients.
\end{abstract}

*Correspondence: deborah.barnes@ucsf.edu

${ }^{2}$ Health Services Research, Mental Health Service, San Francisco VA Medical Center, 4150 Clement Street (151R), San Francisco, CA 94121, USA

Full list of author information is available at the end of the article
There is a growing recognition that the complex pathologic cascade that leads to Alzheimer's disease (AD) begins decades before the development of clinical symptoms [1]. This suggests that effective prevention will require predicting who will develop $\mathrm{AD}$ decades before the onset of symptoms. Therefore, there has been growing interest in developing accurate ways of identifying individuals who have an increased risk of developing symptomatic $\mathrm{AD}$ so that they can be targeted for preventive interventions such as risk factor reduction, behavioral modification, or pharmacologic treatment.

Much of the current work on AD prediction, including the Alzheimer's Disease Neuroimaging Initiative (ADNI), has focused on identifying biomarkers and neuroimaging tests that can accurately detect individuals with preclinical disease. These approaches implicitly recognize the multifaceted nature of the $\mathrm{AD}$ pathologic cascade (amyloid beta deposition, neurofibrillary tangles, neuronal dysfunction, and cerebral volume loss) [2] and rely on different tests to capture different aspects of that pathology. One recent analysis of ADNI data [3] found that a combination of magnetic resonance imaging (MRI), cerebrospinal fluid, and neuropsychological and functional markers predicted conversion from mild cognitive impairment to $\mathrm{AD}$ with moderate accuracy (c statistic $=0.80)$. The c statistic, also known as the area under the receiver operating characteristic curve, may range from 0 to 1 , with 1 reflecting perfect discrimination and 0.5 reflecting no better than chance.

An alternative approach to the prediction of $\mathrm{AD}$ risk has been to identify combinations of traditional risk factors that can reliably differentiate individuals with a high risk of developing $\mathrm{AD}$ from those with a low risk. One study found that mid-life risk factors, including age, education, hypertension, hypercholesterolemia, and obesity, predicted late-life dementia risk with moderate accuracy (c statistic $=0.77$ ) [4]. Another study found that a combination of late-life factors, including age, cognitive test scores, MRI measures, apolipoprotein E (APOE) genotype, cardiovascular disease, and functional measures, also predicted dementia risk with moderate accuracy (c statistic $=0.82)$ [5]. An abbreviated version that included age and simplified measures of cognitive function and cardiovascular disease had moderate accuracy as well $(\mathrm{c}$ statistic $=0.77)[6]$. 
Another recent study extended this work by noting that a tremendous range of factors appear to predict $\mathrm{AD}$, suggesting that AD is more likely in people with a broad decline in health. Song and colleagues [7] found that 19 non-traditional risk factors such as a general health question ('How good is your health?') and sensory questions ('How good is your eyesight or hearing?') predicted which individuals were likely to develop $\mathrm{AD}$, even after accounting for traditional risk factors such as age, sex, education, cognitive function, and cardiovascular disease. Implicit in this study is the assumption that, before leading to symptoms of cognitive impairment, $\mathrm{AD}$ is associated with non-specific symptoms such as worse self-rated health. However, the prognostic accuracy of this model was only fair (c statistic $=0.66$ ). In fact, in another study, demographics alone were found to be more predictive of dementia risk (c statistic $=0.72$ ), and significant increases in prognostic accuracy were associated with the addition of APOE genotype $(\mathrm{c}$ statistic $=0.75)$ and vascular risk factors $(\mathrm{c}$ statistic $=$ 0.79) [8].

Taken together, these studies suggest that there are a variety of approaches for identifying with fair to moderate levels of discrimination those individuals who are at high risk of developing AD. However, all of these models suffer from two important limitations in their approach to the prediction of AD risk.

First, none of these models explicitly accounts for the competing risk of death. AD is predominantly a disease of older adults who experience high rates of death from other causes. To effectively target preventive interventions - in particular, pharmacologic interventions that may have adverse side effects - AD prediction models must identify individuals who are likely to suffer symptomatic AD before death. Many risk factors for AD are also risk factors for mortality (for example, older age, vascular risk factors, and functional limitations) [9]. Therefore, some patients who are at high risk for AD may be at even higher risk for death before symptomatic AD. To appropriately balance the potential benefit of preventive intervention with the potential harm, AD prediction models must account for death and identify individuals whose risk of AD outweighs their risk of death.

Second, dementia risk models developed to date have been developed in cohort studies that focused on a limited range of potential predictors and had relatively short follow-up periods ( $<10$ years) or relatively narrow risk windows (for example, mid-life only and late-life only). Thus, these studies have not examined the full range of AD predictors (biomarkers, neuroimaging techniques, and traditional and non-traditional risk factors) over the full 10 - to 20 -year preclinical risk period. The ideal study would include repeated ascertainment of thousands of individuals over several decades, requiring a tremendous investment in resources. Furthermore, these prospectively collected data would not be available for decades, suggesting that alternative modeling techniques need to be employed. One possible solution is to use hidden Markov models [10], which potentially could be used to combine data from multiple sources to model disease state transitions across the full AD clinicalpathologic spectrum.

In conclusion, prediction of AD risk is a relatively new field of inquiry. Several alternative approaches with moderate levels of accuracy have been developed, but none is ready for widespread clinical use. As the field moves forward, it will be critically important to develop techniques that simultaneously model the risk of mortality as well as the risk of AD over the full preclinical spectrum and to consider the potential harm as well as the benefit of identifying and treating high-risk older patients.

\section{Abbreviations \\ AD, Alzheimer's disease; ADNI, Alzheimer's Disease Neuroimaging Initiative; APOE, apolipoprotein E; MRI, magnetic resonance imaging.}

\section{Competing interests}

The authors declare that they have no competing interests.

\section{Acknowledgments}

This work was supported in part by funding from the S. D. Bechtel, Jr. Foundation, including an Early Investigator award (principal investigator: SJL), and the Program for the Aging Century. SJL was also supported by a Paul Beeson Career Development Award from the National Institute on Aging and the American Federation for Aging Research (K23AG040779). Funders were not involved in the conception of this work, writing of the manuscript, or the decision to submit the manuscript for publication.

\section{Author details}

'Department of Psychiatry, University of California at San Francisco, San Francisco, CA, USA. ${ }^{2}$ Health Services Research, Mental Health Service, San Francisco VA Medical Center, 4150 Clement Street (151R), San Francisco, CA 94121, USA. ${ }^{3}$ Program for the Aging Century, Division of Geriatrics, Department of Medicine, University of California at San Francisco, San Francisco, CA 94118, USA. ${ }^{2}$ Division of Geriatrics, Department of Medicine, University of California at San Francisco, San Francisco, CA 94118, USA. ${ }^{5}$ Health Services Research, Medical Service, San Francisco VA Medical Center, 4150 Clement Street (151R), San Francisco, CA 94121, USA

Published: 25 November 2011

\section{References}

1. Jack CR Jr., Knopman DS, Jagust WJ, Shaw LM, Aisen PS, Weiner MW, Petersen RC, Trojanowski JQ: Hypothetical model of dynamic biomarkers of the Alzheimer's pathological cascade. Lancet Neurol 2010, 9:119-128.

2. Sperling RA, Aisen PS, Beckett LA, Bennett DA, Craft S, Fagan AM, Iwatsubo T, Jack CR Jr., Kaye J, Montine TJ, Park DC, Reiman EM, Rowe CC, Siemers E, Stern Y, Yaffe K, Carrillo MC, Thies B, Morrison-Bogorad M, Wagster MV, Phelps CH: Toward defining the preclinical stages of Alzheimer's disease: recommendations from the National Institute on Aging-Alzheimer's Association workgroups on diagnostic guidelines for Alzheimer's disease. Alzheimers Dement 2011, 7:280-292.

3. Cui Y, Liu B, Luo S, Zhen X, Fan M, Liu T, Zhu W, Park M, Jiang T, Jin JS, Alzheimer's Disease Neuroimaging Initiative: Identification of conversion from mild cognitive impairment to Alzheimer's disease using multivariate predictors. PLoS One 2011, 6:e21896.

4. Kivipelto M, Ngandu T, Laatikainen T, Winblad B, Soininen $H$, Tuomilehto J: Risk score for the prediction of dementia risk in 20 years among middle aged people: a longitudinal, population-based study. Lancet Neurol 2006, 
5:735-741.

5. Barnes DE, Covinsky KE, Whitmer RA, Kuller LH, Lopez OL, Yaffe K: Predicting risk of dementia in older adults: the late-life dementia risk index. Neurology 2009, 73:173-179.

6. Barnes DE, Covinsky KE, Whitmer RA, Kuller LH, Lopez OL, Yaffe K: Commentary on 'Developing a national strategy to prevent dementia: Leon Thal Symposium 2009.' Dementia risk indices: a framework for identifying individuals with a high dementia risk. Alzheimers Dement 2010, 6:138-141.

7. Song X, Mitnitski A, Rockwood K: Nontraditional risk factors combine to predict Alzheimer disease and dementia. Neurology 2011, 77:227-234.

8. Reitz C, Mayeux R, Luchsinger JA: Accuracy of summary risk score for prediction of Alzheimer disease: better than demographics alone?-Reply. Arch Neurol 2011, 68:268-270.

9. Lee SJ, Lindquist K, Segal MR, Covinsky KE: Development and validation of a prognostic index for 4-year mortality in older adults. J Am Med Assn 2006, 295:801-808.

10. Woodbury MA, Manton KG, Yashin Al: Estimating hidden morbidity via its effects on mortality and disability. Stat Med 1988, 7:325-336.

doi:10.1186/alzrt95

Cite this article as: Barnes DE, Lee SJ: Predicting Alzheimer's risk: Why and how? Alzheimer's Research \& Therapy 2011, 3:33. 